\title{
Application of Molecular Probes in Tumor Treatment
}

\author{
Zeyu Chen ${ }^{1, *}$ \\ ${ }^{1}$ School of Materials Science and Chemical Engineering, Ningbo University, Ningbo City, a, 315000, Zhejiang Province, China.
}

\begin{abstract}
Photothermal therapy refers to the addition of targeted drugs with photothermal effects into the patient's body. Through targeting, the drug specifically recognizes tumor cells and accumulates around tumor cells. Afterwards, it is irradiated with a laser of a specific wavelength to achieve a local temperature increase, thereby achieving the effect of killing or locating tumor cells. Therefore, the most important part of this treatment method is the photothermal probe with a targeting effect and good photothermal effect. The existing photothermal probes are mainly composed of inorganic substances, organic small molecules and organic biomolecules. This paper will mainly introduce the development status and prospects of performance of these three types of photothermal probes to introduce photothermal agent development.
\end{abstract}

\section{Introduction}

A tumor refers to the growth of cells formed by local cell proliferation under the action of various tumor-causing factors. Especially malignant tumors have become one of the main causes of death in modern developed countries. Research on tumor treatment has been a popular direction of medical research in recent years, and many tumor treatment methods have been drawn and developed, including chemotherapy, radiotherapy, and surgical treatment. However, traditional tumor treatment methods usually have some drawbacks. Chemotherapy and radiotherapy have low selectivity and have certain side effects. Surgical treatment has a high recurrence rate, and other methods are needed to help locate cancer cells. Therefore, the development direction of modern cancer treatment is mainly to reduce the recurrence rate, reduce side effects, and accurately locate cancer cells. As a kind of photothermal probe developed earlier, inorganic probe has the advantages of relatively mature technology, excellent photothermal performance, and relatively low cost, but it can also cause problems such as heavy metal residues in the body. The second is organic molecules, but this method has disadvantages such as difficulty in accumulation at the tumor site and a relatively poor photothermal effect. In addition, there are organic polymer photothermal materials. This method has the advantages of good biocompatibility, good targeting effect and photothermal effect, and is currently a hot development direction. This paper will mainly analyze the properties, advantages and disadvantages and development status of these three types of photothermal agents.

\footnotetext{
*Corresponding author: 1063714581@qq.com
}

\section{Inorganic optical nanomaterials}

Photothermal therapy requires a material with good photothermal properties as the probe needed to treat tumors at specific locations. The photothermal agent needs to have a good ability to convert light energy into heat energy. Due to the principle of photothermal therapy, the photothermal agent needs to have a targeting effect on cancer cells and the function of specifically recognizing cancer cells. Inorganic nanomaterials, as the earliest developed photothermal agent, have the advantages of excellent photothermal effect and relatively mature technology. The current inorganic photothermal agents mainly include a variety of different structures of gold nanomaterials[1, 2], Pb nanosheets[3], carbon-based nanomaterials[4-6] (mainly including carbon nanotubes, nanographene, carbon nanospheres), Prussian blue[7], copper sulfide nanoparticles[8] and some other emerging nanomaterials (such as quantum dots, transition metals, etc.). These photothermal materials have undergone sufficient development and have been proven by many biomedical work groups to have a good ability to ablate tumor cells.

However, inorganic nanomaterials also have certain limitations. For example, most inorganic nanomaterials are composed of heavy metals (gold nanoparticles, $\mathrm{Pb}$ nanosheets, copper sulfide nanoparticles, etc.), which are difficult to be excreted from the body by normal human metabolism, and after accumulation, they will cause damage to the human body to a certain extent. In addition, the structure of gold nanoparticles has a great influence on their performance. However, after being irradiated by a long-term laser, it can easily change its structure and lose its original function.

Therefore, the main development direction of this type of photothermal materials is to combine with 
polymers to improve their targeting effect on tumor cells, improve their biocompatibility, and reduce their possible harm to human tissues.

\section{Organic small molecule light nanomaterials}

Organic small molecule optical nanomaterials, as a potential alternative to inorganic optical nanomaterials, are due to their good biocompatibility, relatively good photothermal effect and excellent tumor cell targeting effect. It is currently a hot development direction of photothermal nanomaterials. At present, the main research and development of organic photonanomaterials are mainly micelles containing near-infrared dyes (such as cyanine dyes, etc.), porphyrins, and other organic photothermal agents. However, organic small molecule photothermal agents also have their disadvantages. Because it is in a molecular form, it is very easy to be affected by the environment in the organism, causing degradation and other problems. As a result, it is more difficult to accumulate at the tumor site. In addition, most of the organic small molecule photothermal agents have the ability to convert light energy into heat, and they also have fluorescence characteristics, which will compete with the photothermal effect and reduce the photothermal conversion efficiency of organic small molecule photothermal agents. However, in recent years, by optimizing the structure of organic small molecule photothermal agents, its stability and photothermal conversion efficiency have been greatly improved

\subsection{Micelles containing near-infrared dyes}

In recent years, near-infrared dyes have been widely used in the field of fluorescence imaging. After research, it was found that after being excited, this type of dye not only emits fluorescence, but also converts part of the light energy into heat energy. Among them, cyanine dyes such as indocyanine green (ICG) and cypate can convert light energy to heat energy instead of fluorescence to the maximum extent. Therefore, the current research on photothermal agents based on cyanine dyes is a hot topic direction. However, due to its structural characteristics, its stability in aqueous solution is poor, its photostability is poor, and it is difficult to gather near the tumor. Therefore, some nanomolecules are commonly adopted to modify it. The modified cyanine dyes have better solubility stability and light stability. For example, by modifying the end groups of cyanine dyes to be hydrophilic, while retaining the hydrophobic head, in this way, in the water environment in the body, the molecules will self-assemble to form a group, thereby reducing the damage on body environment.

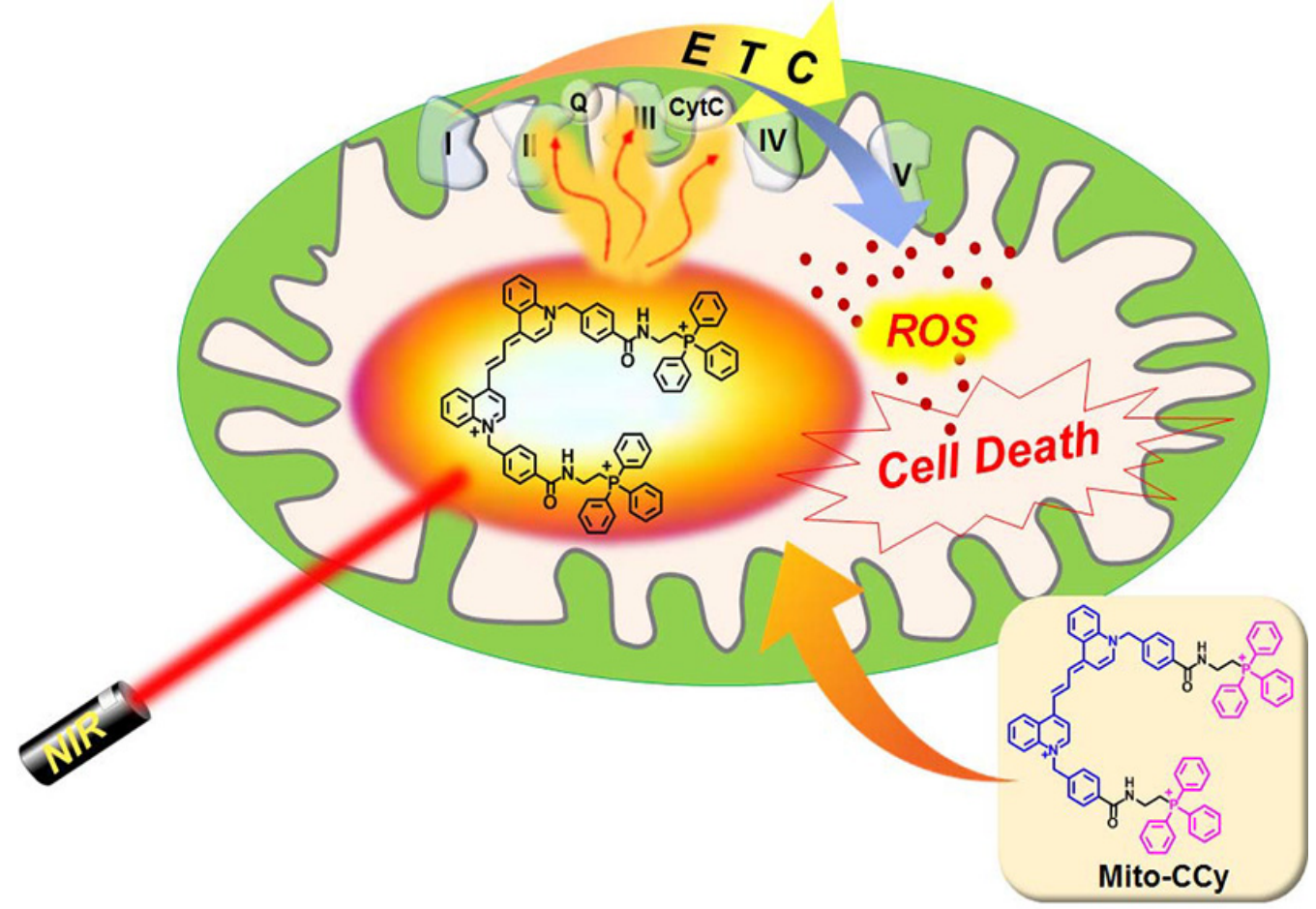

Fig. 1. Mito-CCy's photothermal treatment of tumor cells

Indocyanine green has excellent photothermal conversion efficiency and strong light absorption characteristics. As an excellent photothermal agent, it has been approved by the U.S. Food and Drug Administration (FDA) for direct use in disease diagnosis[9]. In 2017, based on the cyanine probes, Kim's group designed a photosensitizer (Mito-CCy) that targets organelles and has high light-to-heat conversion efficiency (as presented in Figure 1) [10]. The photosensitizer not only has good biocompatibility and phototoxicity, but also achieves the targeting effect on organelles by modifying the triphenylphosphine functional group with the mitochondrial targeting effect on the photothermal agent. 


\subsection{Porphyrins}

Porphyrins are heterocyclic compounds with four pyrrole subunits bridged by methine groups, and are currently widely employed in photothermal therapy and imaging. However, the monomer structure porphyrin also has the common disadvantage of organic small molecule photothermal agents, that is, it is difficult to accumulate and accumulate at the tumor site. In addition, its water solubility is also poor and it is difficult to utilize in the body. In order to solve this problem, the main method now is to carry out supramolecular assembly through intermolecular forces. Through supramolecular assembly, the drugs or photothermal agents are assembled separately or together with other light-to-heat conversion nanomaterials to form an overall nanosystem platform [11].

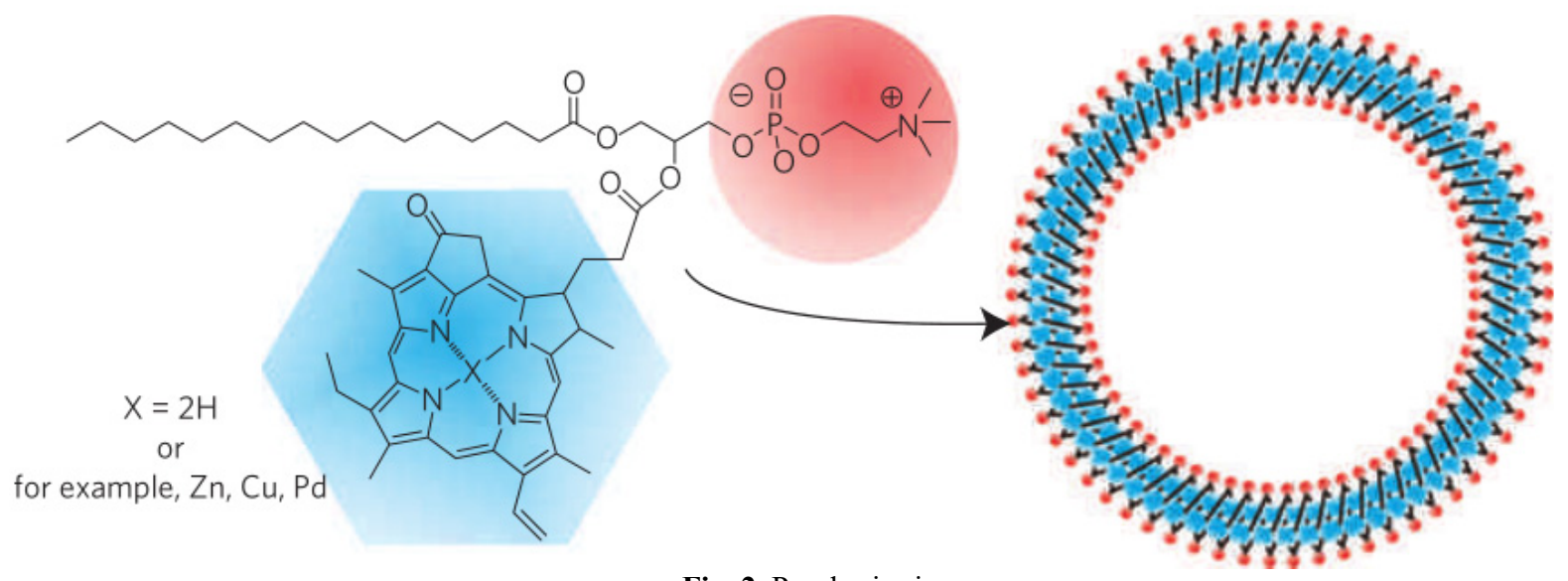

Fig. 2. Porphyrin rings

Lovell et al reported for the first time that porphyrin rings can be used for photothermal treatment of tumors(as shown in Figure 2)[12]. They found that porphyrins and phospholipids can self-assemble into a liposome structure. This structure not only has a high porphyrin loading, but also excellent biocompatibility and NIR absorption capacity.

However, most of the absorption wavelengths of porphyrins currently used in photothermal treatment are short wavelengths of $600-700 \mathrm{~nm}$, which limits their application in organisms. Therefore, the current development direction is to design a wavelength that can absorb wavelengths above $800 \mathrm{~nm}$. Porphyrin photothermal agent with strong near-infrared radiation.

\subsection{Other small organic molecules}

In addition to common cyanine dyes and porphyrins, there are also some other small organic molecules that have the ability to convert light to heat. For example, phthalocyanine with good molar absorbance and optical stability, which is often used in photodynamic therapy, has also begun to be emplyed in photothermal therapy in recent years. $\mathrm{Li}$ et al prepared a zinc phthalocyanine compound whose terminal hydroxyl group can be modified by biotin[13]. The phthalocyanine compound can easily self-assemble into nanoparticles with a diameter of 100-200 nm. After experimental research, it is found that the phthalocyanine particles with this specific structure can accumulate in the A549 tumors of xenotransplanted mice after being injected into mice, and show the synergy of photodynamic therapy and photothermal therapy after receiving NIR light irradiation. The therapeutic effect obviously inhibits the growth of tumors. In addition, in 2016, Dong et al. synthesized a small organic molecule with a D-A-D (donor-acceptor-donor) structure for photoacoustic imaging-mediated photodynamic (PDT)/photothermal (PTT) co-therapy [14]. This small organic molecule exhibits high photothermal conversion efficiency and excellent singlet oxygen yield under $660 \mathrm{~nm}$ laser irradiation. In addition, it also exhibits excellent tissue penetration ability, and has shown a good tumor treatment effect in mouse experiments.

\section{Organic polymer photothermal materials}

At present, the photothermal agents developed based on organic polymer materials are mainly synthetic semiconducting polymer nanoparticles (SPNPs)[15]. SPNPs have high absorption coefficient, adjustable light absorption range, high light stability and good biocompatibility. The first organic polymer photothermal agent reported to be used in photothermal therapy is polyaniline (PANI). However, until now, only a small number of PANI can be applied as photothermal agents. This may be due to PANI doping later, the structure became more complicated, which affected its photothermal properties. In 2018, Lyu et al prepared a water-soluble SPNPs with good biodegradability and photothermal properties[16]. The vinyl bonds of such SPNPs can be involuted in nanoparticles, thereby increasing the absorption coefficient per unit mass. And light-to-heat conversion efficiency. Such SPNPs can not only provide signals for photoacoustic imaging, but also can thermally ablate tumors in mice while detecting. In addition, since such SPNPs can undergo enzymatic degradation in the presence of oxidizing substances, the risk of long-term toxicity caused by accumulation of the substances in the body is avoided. 


\section{Conclusion}

At present, photothermal therapy has performed well in the field of tumor treatment due to its minimally invasive and non-invasive characteristics. And it has been proven to have good anti-cancer effects in preliminary clinical trials. In addition, the rapid development of nanotechnology has provided a variety of high-efficiency photothermal agents for photothermal therapy. This study mainly introduces various current nanomaterials for photothermal conversion, and lists the current defects of each material. In order to improve the effect of photothermal therapy, it can be achieved by designing more efficient photothermal conversion agents. Of course, in addition, it is necessary to control local heat generation so that it can kill tumor cells without affecting normal human cells. In addition, the heat generation of the photothermal agent can be precisely controlled by improving the incident laser light.

However, the current photothermal therapy is still facing the problem that the diversity, complexity and heterogeneity of tumors make it difficult to completely eliminate the entire tumor by photothermal therapy alone, and it is difficult to prevent tumor recurrence and metastasis. The current development direction is to treat tumors by combining photothermal therapy with other treatment methods. In addition, the safety of photothermal agents remains to be studied, because most photothermal agents are currently obtained through more complicated preparation methods, which may cause some toxic intermediates to enter the human body and cause long-term toxicity. However, if the biodegradability of the photothermal agent is improved, it will affect the retention time of the photothermal agent in the blood and the accumulation of the photothermal agent at the tumor site. In addition, the complicated preparation methods and raw materials of photothermal agents have also led to the high cost of photothermal treatment. These are the problems that need to be solved at the current stage.

As a result, although photothermal therapy still has some drawbacks to be solved by research, it provides a new development direction for tumor treatment, and with the development of material technology, the current drawbacks may also be overcome in the future. Therefore, photothermal therapy is a tumor treatment with good development prospects.

\section{Acknowledgements}

I would like to express my gratitude to all those who helped me during the write of this paper.

First of all, I want to express my gratitude to professor Wenying Li. She gave me some suggestions on the topic and main structure of my paper. Without her patient help, this paper would be difficult to complete.

Second, I want to appreciate Min Han. She helped to correct some detailed errors in my paper, and gave me some advice of how to make this paper more complete.

Finally, I want to thank Jianyu Ma. He gave me some suggestions on detailing the topic of the paper, and also gave me many useful suggestions in the writing of the paper.

\section{References}

1. Nikoobakht B, El-Sayed M A. Preparation and growth mechanism of gold nanorods (NRs) using seed-mediated growth method[J]. Chem Mater, 2003, 15(10): 1957-1962.

2. Meng L J, Niu L Y, Li L, et al. Gold nanoparticles grown on ionic liquid-functionalized single-walled carbon nanotubes: new materials for photothermal therapy[J]. Chem Eur J, 2012, 18(42): 13314-13319.

3. Fang W, Yang J, Gong J, et al. Photo- and $\mathrm{pH}$-triggered release of anticancer drugs from mesoporous silica-coated $\mathrm{Pd} @ \mathrm{Ag}$ nanoparticles[J]. Adv Funct Mater, 2012, 22(4): 842-848.

4. Robinson J T, Tabakman S M, Liang Y Y, et al. Ultrasmall reduced graphene oxide with high near-infrared absorbance for photothermal therapy[J]. J Am Chem Soc, 2011, 133(17): 6825-6831.

5. Meng L J, Xia W J, Liu L, et al. Golden single-walled carbon nanotubes prepared using double layer polysaccharides bridge for photothermal therapy[J]. ACS Appl Mater Interfaces, 2014, 6(7): 4989-4996.

6. Cheng L, Wang C, Feng L Z, et al. Functional nanomaterials for phototherapies of cancer[J]. Chem Rev, 2014, 114(21): 10869-10939.

7. Chen W S, Zeng K, Liu H, et al. Cell membrane camouflaged hollow Prussian blue nanoparticles for synergistic photothermal-/chemotherapy of cancer[J]. Adv Funct Mater, 2017, 27(11): 1605795.

8. Wu L, Wu M, Zeng Y, et al. Multifunctional PEG modified DOX loaded mesoporous silica nanoparticle@CuS nanohybrids as photo-thermal agent and thermal-triggered drug release vehicle for hepatocellular arcinoma treatment[J]. Nanotechnology, 2015, 26(2): 025102.

9. Sheng Z H, Hu D H, Xue M M, et al. Indocyanine green nanoparticles for the ranostic applications[J]. Nano-micro Letters, 2013, 5(3): 145-150.

10. Jung, H. S.; Lee, J.-H.; Kim, K.; Koo, S.; Verwilst, P.; Sessler, J.; Kang, C.; Kim, J. S. A Mitochondria-Targeted Cryptocyanine-Based Photothermogenic Photosensitizer. [J]. J. Am. Chem. Soc. 2017, 139(29): 9972-9978.

11. Zhao L Y, Liu Y M, Chang R, et al. Supramalecular photothermal nanomaterials as an emerging paradigm toward precision cancer therapy[J]. Advanced Functional Materials, 2019, 29(4): 1806877.

12. Lovell J F, Jin C S, Huynh E, et al. Porphysome nanovesicles generater by porphyrin bilayers for use as multimodal biophotonic contrast agents[J]. Nature Materials, 2011, 10(4): 324-332.

13. Li X S, Kim C, Lee S A, et al. Nanostructured phthalocyanine assemblies with protein-driven 
switchable photoactivities for biophotonic imaging and therapy[J]. Journal of the American Chemical Society, 2017, 137(31): 10880-10886

14. Cai, Y.; Liang, P.; Tang, Q.; Yang, X.; Si, W.; Huang, W.; Zhang, Q.; Dong, X.

Diketopyrrolopyrrole-Triphenylamine Organic Nanoparticles as Multifunctional Reagents for Photoacoustic ImagingGuided

Photodynamic/Photothermal Synergistic Tumor Therapy. [J]. ACS Nano 2017, 11(1): 1054-1063.

15. Vines J B, Lim D, Park H. Contemporary polymerbased nanoparticle systems for photothermal therapy[J]. Polymers, 2018, 10(12): 1357

16. Lyu Y, Zeng J F, Jiang Y Y, et al. Enhancing both biodegradability and efficacy of semiconducting polymer nanoparticles for photoacoustic imaging and photothermal therapy[J]. ACS Nano, 2018, 12(2): 1801-1810. 